\title{
Melatonin inhibits vascular smooth muscle cell proliferation and apoptosis through upregulation of Sestrin2
}

\author{
SEUNGHYEONG LEE ${ }^{1,2^{*}}$, JUN-KYU BYUN ${ }^{3,4^{*}}$, MIHYANG PARK $^{1,2}$, SUNG WOO KIM $^{5}$, SUNGWOO LEE ${ }^{6}$, \\ JUNG-GUK KIM ${ }^{4}$, IN-KYU LEE ${ }^{4}$, YEON-KYUNG CHOI ${ }^{4}$ and KEUN-GYU PARK ${ }^{1,3,4}$
}

\author{
${ }^{1}$ Department of Biomedical Science, Graduate School; ${ }^{2}$ BK21 Plus KNU Biomedical Convergence Program; \\ ${ }^{3}$ Research Institute of Aging and Metabolism, Kyungpook National University, Daegu 41566; ${ }^{4}$ Department of Internal Medicine, \\ School of Medicine, Kyungpook National University, Kyungpook National University Hospital, Daegu 41944; ${ }^{5}$ Department of \\ Internal Medicine, Catholic University of Daegu School of Medicine, Daegu 42472; ${ }^{6}$ New Drug Development Center, \\ Daegu-Gyeongbuk Medical Innovation Foundation, Daegu 41061, Republic of Korea
}

Received June 25, 2019; Accepted December 10, 2019

DOI: $10.3892 /$ etm.2020.8638

\begin{abstract}
Excessive vascular smooth muscle cell (VSMC) proliferation contributes to the development of atherosclerosis and restenosis. Furthermore, apoptosis of VSMCs accelerates plaque rupture in the atherosclerotic vessels. Therefore, a strategy that regulates both VSMC proliferation and apoptosis is essential for the development of novel pharmacological tools for the treatment of atherosclerosis. Despite mounting evidence supporting the benefits of melatonin in diverse metabolic diseases, the role of melatonin in VSMC growth remains largely unknown. The present study revealed that melatonin inhibited both proliferation and apoptosis of primary cultured rat VSMCs. Melatonin induced mitochondrial energetic stress in VSMCs and subsequent induction of Sestrin2 via C/EBP $\beta$. Melatonin-induced Sestrin2 suppressed mTORC1 activity in VSMCs, contributing to suppression of VSMC proliferation. Additionally, melatonin-induced upregulation of Sestrin2 blocked apoptosis by preventing excessive ROS generation. The results demonstrated that melatonin controlled VSMC proliferation and apoptosis via Sestrin2-mediated inhibition of mTORC1 and ROS scavenging. Therefore, melatonin should be considered as a lead compound for therapies aimed at preventing vessel lumen constriction during the course of atherosclerosis and restenosis.
\end{abstract}

Correspondence to: Dr Keun-Gyu Park or Dr Yeon-Kyung Choi, Department of Internal Medicine, School of Medicine, Kyungpook National University, Kyungpook National University Hospital, 130 Dongdeok-ro, Jungu, Daegu 41944, Republic of Korea

E-mail:kpark@knu.ac.kr

E-mail: exc4932@hanmail.net

*Contributed equally

Key words: vascular smooth muscle cells, melatonin, Sestrin2, proliferation, apoptosis

\section{Introduction}

Vascular smooth muscle cells (VSMCs) are the predominant cells in arteries (1). Excessive VSMC proliferation and neo-intima formation are critical processes in the development of atherosclerosis and restenosis after percutaneous balloon angioplasty (2). In addition to VSMC growth, proper regulation of apoptosis of VSMCs within vessel walls is also important for preventing plaque rupture in atherosclerotic vessels (3). For these reasons, many studies have focused on the regulation of VSMC growth and apoptosis by mechanistic target of rapamycin complex 1 (mTORC1) $(4,5)$. It is well established that mTORC1 contributes to vascular pathologies such as intimal hyperplasia; accordingly, the use of the mTORC1 inhibitor rapamycin in drug-eluting stents has achieved a profound reduction in the incidence of restenosis (5).

Sestrin 2 is a stress-inducible protein that regulates cell growth and survival, and can participate in cellular responses to stress conditions (6). Sestrin2 suppresses mTORC1 signaling via activation of AMPK-activated protein kinase (AMPK) or inhibition of Rag GTPases $(7,8)$. Through these functions, Sestrin 2 attenuates oxidative stress, fat accumulation, and insulin resistance, thereby attenuating various age- and obesity-related metabolic diseases (6). Sestrin 2 is also induced by a range of mitochondrial dysfunctions and promotes mitochondrial biogenesis and mitohormesis (9). In the liver, Sestrin2 expression is induced upon chronic hypernutrition and acts as an important suppressor of hepatosteatosis by inhibiting mTORC1 (10). Despite a great deal of evidence supporting the idea that Sestrin 2 inhibits mTORC1 in the context of metabolic pathologies, the effect of Sestrin2/mTORC1 on VSMC growth has not been previously studied.

Melatonin was first described as an endogenous hormone that regulates circadian and seasonal rhythms. Several lines of evidence have revealed that melatonin has many other functions, including roles as an antioxidant, immune enhancer, promoter of mitochondrial homeostasis, and tumor suppressor (11). Multiple mechanisms, including cell cycle arrest and epithelial-to-mesenchymal transition, are thought to mediate the biologic effects of melatonin on growth 
and invasion of cancer (12). In addition, melatonin blocks TGF- $\beta 1$-induced fibroblast proliferation, which leads to alleviated pulmonary fibrogenesis (13). However, it remains unclear whether melatonin regulates VSMC growth and proliferation. In this study, we investigated whether melatonin modulates VSMC growth and, if so, whether Sestrin2/mTORC1 is implicated in this effect.

\section{Materials and methods}

Cell culture. Rat aortic smooth muscle cells were isolated from 4-week-old male Sprague-Dawley rats (90 to $100 \mathrm{~g}$ ). Cervical dislocation euthanasia was performed by well-trained individuals. Trimmed aortas was washed with sterilized cold phosphate-buffered saline (PBS) and sliced into pieces measuring 1-3 $\mathrm{mm}^{2}$. The pieces of aorta were attached to dishes and cultured in low-glucose Dulbecco's modified Eagle's medium (Hyclone) supplemented with $20 \%$ fetal bovine serum (FBS; Hyclone) for 2 weeks at $37^{\circ} \mathrm{C}$ in $5 \%$ $\mathrm{CO}_{2}$. The medium was replaced every day. Cells were maintained in low-glucose Dulbecco's modified Eagle's medium supplemented with $20 \%$ FBS. Cells at passage 4-9 were used in all experiments. All protocols for animal use and euthanasia were reviewed and approved by the Animal Care and Use Committee of Kyunpook National University School of Medicine and conducted according to our institutional guideline (KNU-2011-0096-1).

Western blot analysis. Cells were lysed in lysis buffer $(20 \mathrm{mM}$ Tris-HCl [pH 7.4], $5 \mathrm{mM}$ EDTA [pH 8.0], $10 \mathrm{mM} \mathrm{Na}_{4} \mathrm{P}_{2} \mathrm{O}_{7}$, $100 \mathrm{mM} \mathrm{NaF}, 2 \mathrm{mM} \mathrm{Na} \mathrm{VO}_{4}, 1 \% \mathrm{NP}-40$ ) containing aprotinin, leupeptin, PMSF, and phosphatase inhibitors cocktail 3 (Sigma). Protein samples were separated on 10\% SDS-PAGE gels and transferred to PVDF membranes. Membranes were blocked with 5\% skim milk in Tris-buffered saline containing $0.1 \%$ Tween 20 (TBST) and incubated with primary antibody overnight at $4^{\circ} \mathrm{C}$. Primary antibodies against the following proteins were obtained from the indicated suppliers: $\mathrm{C} / \mathrm{EBP} \beta$, Sestrin2, phospho-p70S6K (T389), p70S6K, p-Rb (S807/811) were from Cell Signaling Technology; Rb was from Santa Cruz Biotechnology; and $\beta$-actin (1:5,000) from Sigma.-Membranes were washed three times with TBST and incubated with HRP-conjugated anti-mouse (Santa Cruz Biotechnology) or anti-rabbit secondary antibody (Cell Signaling Technology). HRP was detected using the ECL reagent (BioNote).

Small interfering RNA (siRNA) transfection. For gene silencing, cells were transfected with $50 \mathrm{nM}$ scrambled siRNA, siSestrin2, or siCEBP/ $\beta$ (Bioneer,) using Lipofectamine RNAiMAX (Invitrogen).

Transfection of expression vectors. For overexpression of Sestrin2, cells were transfected with a FLAG-Sestrin2 expressing vector (a gift from Dr. Jun Hee Lee, University of Michigan) or a control vector using TransIT-LT1 Transfection Reagent (Mirus Bio) according to the manufacturer's instructions.

Cell counting. Primary VSMCs transfected with scrambled small interfering RNA (siRNA) or siRNA targeting Sestrin2
(siSestrin2) for $24 \mathrm{~h}$ were serum-starved for $24 \mathrm{~h}$ and incubated with $10 \%$ FBS with or without $2 \mathrm{mM}$ melatonin (Sigma). Cells were trypsinized, stained with Trypan blue solution, and counted with a hemocytometer.

Cell viability assay. Cells were seeded at $3-5 \times 10^{3}$ cells per well in 96-well plates. The cells were serum-starved for $24 \mathrm{~h}$ and incubated with $10 \%$ FBS with or without $2 \mathrm{mM}$ melatonin for 3 days, and cell viability was measured using a CCK8 Solution Reagent (CK04; Dojindo). The absorbance of each well at $495 \mathrm{~nm}$ was measured on a VERSA MAX ELISA reader (Molecular Devices). The proportion of viable cells in each treatment group was normalized against that of control wells.

Flow cytometric analysis. Cell cycle distribution of VSMCs after treatment with $10 \%$ FBS with or without $2 \mathrm{mM}$ melatonin was detected by flow cytometry. VSMCs were collected and fixed in $70 \%$ ethanol at $4^{\circ} \mathrm{C}$ for $30 \mathrm{~min}$. Before analysis, cells were centrifuged at $2000 \mathrm{rpm}$ for $5 \mathrm{~min}$ to remove ethanol. Then, cells were suspended in $500 \mu \mathrm{l}$ PBS containing propidium iodide $(33 \mu \mathrm{g} / \mathrm{ml})$ and Ribonuclease $\mathrm{A}(1 \mathrm{mg} / \mathrm{ml})$ in darkness at $37^{\circ} \mathrm{C}$ for $30 \mathrm{~min}$. Fluorescence emitted by PI-DNA complexes was measured on an Epics XL flow cytometer (BD Bioscience).

Measurement of oxygen consumption rate. Oxygen consumption rate (OCR) was measured in 24-well plates using a Seahorse XF-24 analyzer (Seahorse Bioscience). VSMCs were serum-starved for $24 \mathrm{~h}$ and incubated with $10 \%$ FBS with or without melatonin $(2 \mathrm{mM})$. On the day before the experiment, the sensor cartridge was placed in calibration buffer supplied by Seahorse Bioscience and incubated at $37^{\circ} \mathrm{C}$ in a non- $\mathrm{CO}_{2}$ incubator for $1 \mathrm{~h}$. Oligomycin (Sigma), carbonyl cyanide 3-chlorophenylhydrazone (CCCP, Sigma), and rotenone (Sigma) were added at the indicated times during OCR measurement.

Reverse transcription-quantitative PCR. Total RNA was prepared using QIAzol lysis reagent (Qiagen), and complementary DNA (cDNA) was synthesized from total RNA using the RevertAid First Strand cDNA Synthesis kit (Thermo Fisher Scientific, Inc.). The resultant cDNA was amplified on a 7500 Fast Real-Time PCR System (Applied Biosystems). Relative expression levels were calculated using the $\Delta \Delta \mathrm{Cq}$ method (14); the levels of each mRNA were normalized against the corresponding level of $36 B 4 \mathrm{mRNA}$ primer, and the sequences were as follows: $C E B P \beta$ forward, ACGAGCGGCTGCAGAAGA, reverse, GGCAGCTGCTTGAACAAGTTC; SESN2 forward, ACCTTTCGTGCCCAGGATTAT, reverse, GGGTAGAGC CGCTGGATCA; $36 B 4$ forward, TTCCCACTGGCTGAA AAGGT, and reverse, GCCGCAGCCGCAAA.

MitoSox. Mitochondrial reactive oxygen species (ROS) generation was assessed using MitoSOX Red Mitochondrial Superoxide indicator (Invitrogen). VSMCs transfected with $50 \mathrm{nM}$ scrambled siRNA or siSestrin 2 for $24 \mathrm{~h}$ on a confocal dish were serum-starved for $24 \mathrm{~h}$ and incubated with $10 \%$ FBS with or without $2 \mathrm{mM}$ melatonin. Cells were treated with $5 \mu \mathrm{M}$ MitoSOX reagent working solution and incubated for $10 \mathrm{~min}$ at $37^{\circ} \mathrm{C}$ in the dark. The cells were then washed gently three times with warm HBSS buffer. Finally, cells were 

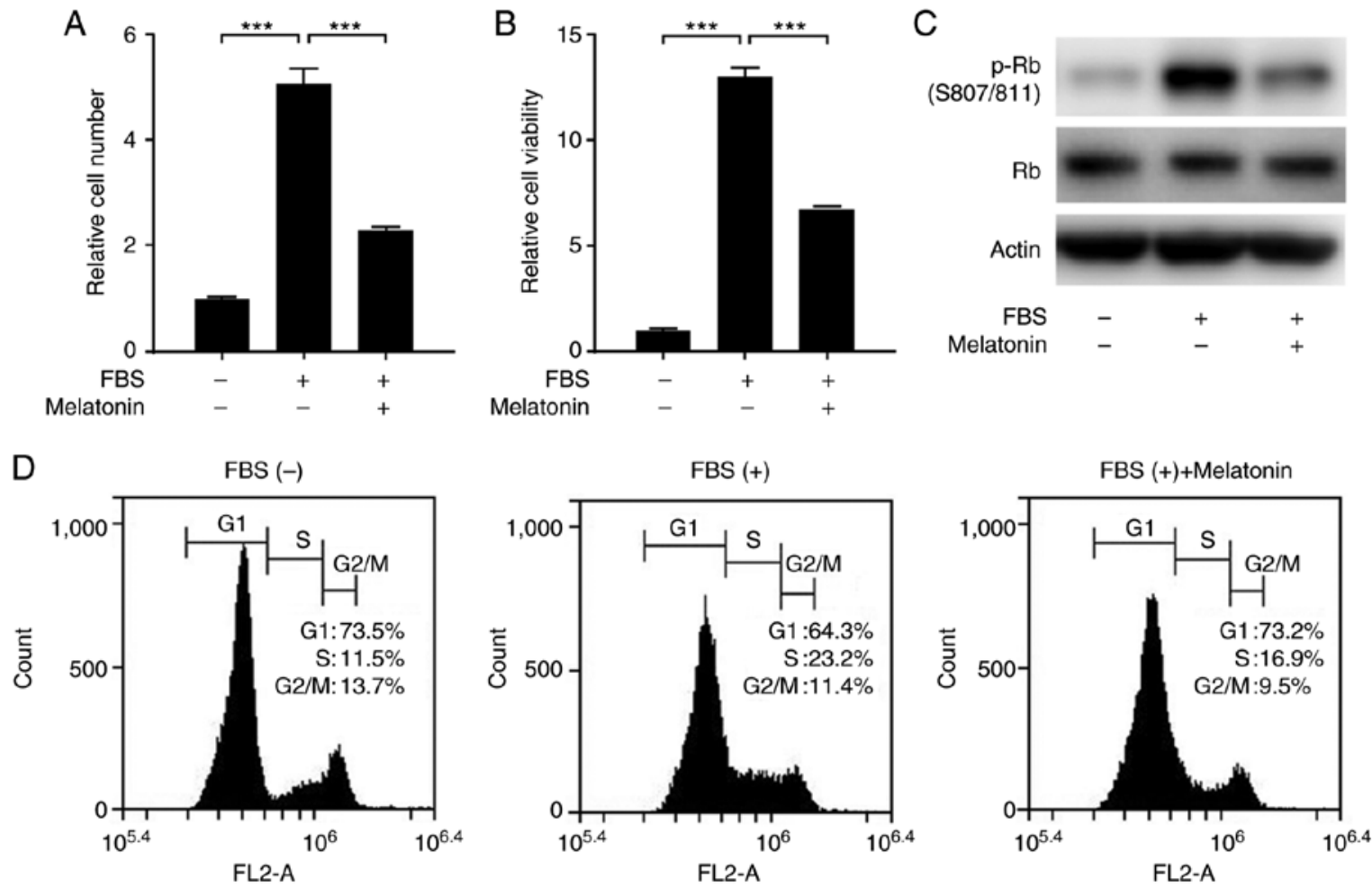

Figure 1. Effects of melatonin on VSMC proliferation. Primary rat VSMCs were serum-starved for $24 \mathrm{~h}$, and then treated with $10 \%$ FBS with or without $2 \mathrm{mM}$ melatonin for $24 \mathrm{~h}$. Relative (A) cell number and (B) cell viability showing the effect of melatonin in FBS-stimulated VSMCs. Data are presented as the mean $\pm \operatorname{SEM}(n=3) .{ }^{* * *} \mathrm{P}<0.001$. Quiescent cells were treated with $10 \%$ FBS with or without $2 \mathrm{mM}$ melatonin. (C) Phosphorylated Rb levels in primary rat VSMCs. (D) Representative flow cytometric data derived from analysis of cell cycle progression in VSMCs. VSMC, vascular smooth muscle cell; p-, phosphorylated; $\mathrm{Rb}$, retinoblastoma protein.

counterstained with NucBlue Live Cell Stain ReadyProbes (Invitrogen) and mounted in warm buffer for imaging. MitoSOX fluorescence intensity were quantified using Image J software.

Immunocytochemistry. Cells were pretreated with or without $2 \mathrm{mM}$ melatonin for $24 \mathrm{~h}$ and then treated with $1 \mathrm{mM} \mathrm{H}_{2} \mathrm{O}_{2}$ for $6 \mathrm{~h}$. Cells were fixed with $4 \%$ paraformaldehyde (Biosesang) and washed with PBS. Cells were permeabilized with $0.1 \%$ Triton X-100 for 15 min and washed with PBS. Following $1 \mathrm{~h}$ of blocking in 5\% normal goat serum (Vector Laboratories) in PBS, cells were incubated with primary anti-cleaved caspase-3 (1:400; Cell Signaling Technology) antibody overnight at $4^{\circ} \mathrm{C}$. After washing with PBS, the cells were incubated with Alexa Fluor ${ }^{\circledR} 568$ goat anti-rabbit (1:100; Thermo Fisher Scientific Inc.) secondary antibodies for $2 \mathrm{~h}$ at room temperature. Nuclei were stained with DAPI (Vector Laboratories). Immnofluorescence intensity of cleaved caspase-3 was quantified using Image $\mathbf{J}$ software.

Statistical analysis. All values are presented as means \pm SEM. ANOVA was used for comparisons between multiple groups, followed by Tukey's post hoc test. $\mathrm{P}<0.05$ was considered to indicate a statistically significant difference.

\section{Results}

Melatonin inhibits VSMC proliferation. We first examined the effect of melatonin on FBS-stimulated proliferation of VSMCs. Treatment of VSMCs with FBS significantly increased the proliferation and viability of VSMCs, but this effect was blocked by melatonin (Fig. 1A and B). Next, we explored whether melatonin inhibits cell cycle progression in VSMCs. We found that melatonin reduced the level of phosphorylated retinoblastoma protein (p-Rb) (Fig. 1C). Flow cytometric analysis of cell cycles showed that melatonin attenuated serum-stimulated progression from $\mathrm{G} 1$ to $\mathrm{S}$ phase. In the melatonin-treated samples, the cells accumulated in G1 phase $(73.2 \%$ in melatonin-treated cells vs. $64.3 \%$ in control cells) with a concomitant decrease in the percentage of cells in S phase (16.9\% in melatonin-treated cells vs. $23.2 \%$ in control cells). Thus, melatonin arrests cells at the G1 cell cycle phase, blocking proliferation (Fig. 1D).

Melatonin induces mitochondrial energetic stress and Sestrin2 expression in VSMCs. To determine the mechanism by which melatonin inhibits VSMC proliferation, we evaluated the effect of melatonin on mitochondrial function using an XF analyzer. We measured basal OCR over time, and then observed the effects of the mitochondrial inhibitors oligomycin, CCCP, and rotenone. Treatment of VSMCs with melatonin caused a significant decrease in the major parameters of mitochondrial function, including basal OCR and maximal respiration, as well as ATP-linked respiration (Fig. 2A-D). After demonstrating that melatonin induced mitochondrial dysfunction, we evaluated CCAAT/enhancer binding protein $\beta$ (C/EBP $\beta)$ and Sestrin2, which promote the response to mitochondrial stress $(15,16)$. As shown in Fig. 3A and B, treatment of VSMCs 

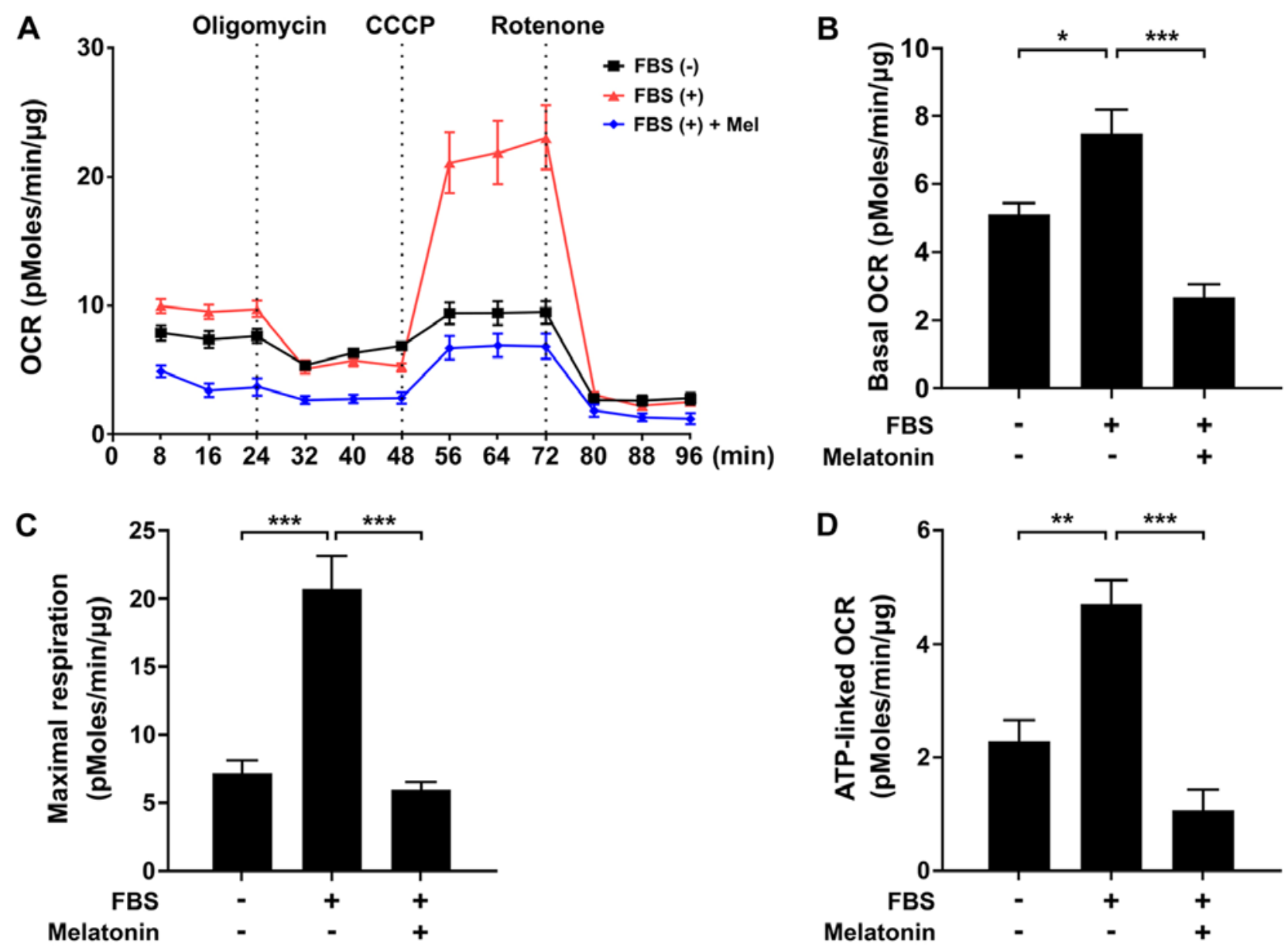

Figure 2. Effects of melatonin on mitochondrial oxygen consumption rate and ATP production in VSMCs. (A) Mitochondrial oxygen consumption rate in FBS-stimulated primary rat VSMCs treated with or without melatonin for $24 \mathrm{~h}$, following exposure to oligomycin $(1 \mu \mathrm{M})$, CCCP $(2 \mu \mathrm{M})$ and rotenone $(1 \mu \mathrm{M})$. (B) Rates of basal respiration, (C) maximal respiratory capacity and (D) ATP-linked respiration in VSMCs shown in (A). Data were normalized against protein content and are presented as the mean $\pm \operatorname{SEM}(n=4) . \quad \mathrm{P}<0.05 ;{ }^{* *} \mathrm{P}<0.01 ;{ }^{* * *} \mathrm{P}<0.001$. VSMC, vascular smooth muscle cell; OCR, oxygen consumption rate; CCCP, carbonyl cyanide 3-chlorophenylhydrazone.

with melatonin increased $\mathrm{C} / \mathrm{EBP} \beta$ and Sestrin2 protein levels. Furthermore, siRNA-mediated knockdown of C/EBP $\beta$ inhibited melatonin-induced upregulation of Sestrin 2 mRNA and protein level (Fig. 3C-E). Taken together, these data demonstrate that melatonin-induced mitochondrial energetic stress results in upregulation of Sestrin2 via C/EBP $\beta$.

Melatonin-induced Sestrin2 prevents VSMC apoptosis. Next, we sought to confirm that Sestrin 2 negatively regulates mTORC1 activity in VSMCs, consistent with previous results $(7,8,17)$. As mTORC1 controls cell growth via direct phosphorylation of Thr389 in the hydrophobic motif site of p70S6 kinase 1 (S6K1), we determined mTORC1 activity by measuring the levels of S6K (T389) phosphorylation (18). The results showed that melatonin increased Sestrin2 but decreased the levels of S6K (T389) phosphorylation; this effect was abolished by siRNA-mediated knockdown of Sestrin2 (siSESN2) in VSMCs (Fig. 4A). Successful knockdown of Sestrin2 was confirmed by quantitative PCR (Fig. 4B). Given that melatonin-induced Sestrin 2 suppressed mTORC1 activity, we next asked whether inhibition of Sestrin2 would restore melatonin-induced suppression of VSMC proliferation. Unexpectedly, siSESN2 further decreased the number of VSMCs beyond the reduction induced by melatonin treatment (Fig. 4C). Because Sestrin2 functions as a ROS scavenger (19), we investigated whether the siSESN2-induced decrease in cell number was caused by cell death triggered by intracellular ROS production. Indeed, siSESN2 significantly increased mitochondria-derived superoxide production (stained by MitoSOX) and the level of cleaved caspase 3 in melatonin-treated VSMCs (Fig. 4D and E). Moreover, Sestrin 2 overexpression decreased mTORC1 activity in VSMCs and the level of cleaved caspase 3 in $\mathrm{H}_{2} \mathrm{O}_{2}$-treated VSMCs, further confirming the anti-proliferative and -apoptotic function of Sestrin2 (Fig. 4F and G). Finally, immunofluorescence using a cleaved caspase 3 -specific antibody showed that melatonin reduced $\mathrm{H}_{2} \mathrm{O}_{2}$-induced apoptosis of VSMCs (Fig. $4 \mathrm{H}$ ). Collectively, these data indicated that melatonin-induced Sestrin 2 not only decreases VSMC proliferation, but also blocks apoptosis of VSMCs by preventing excessive ROS generation.

\section{Discussion}

The results of this study demonstrate that melatonin induces mitochondrial energetic stress in VSMCs, leading to induction of Sestrin2 via C/EBP $\beta$. Melatonin-induced upregulation of Sestrin 2 suppressed mTORC1 activity in VSMCs, contributing 
A

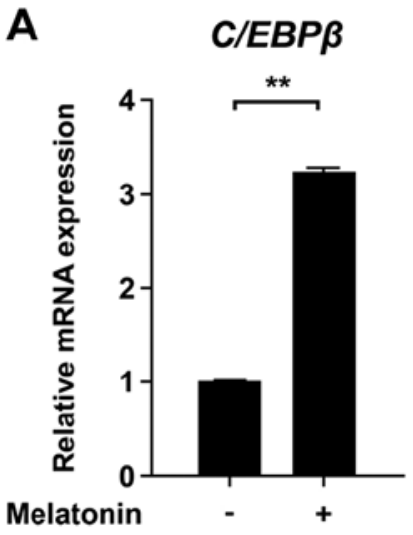

B

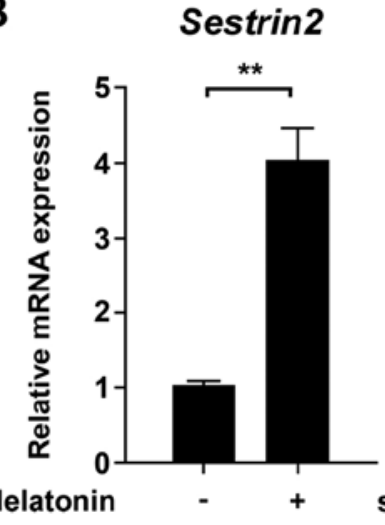

C

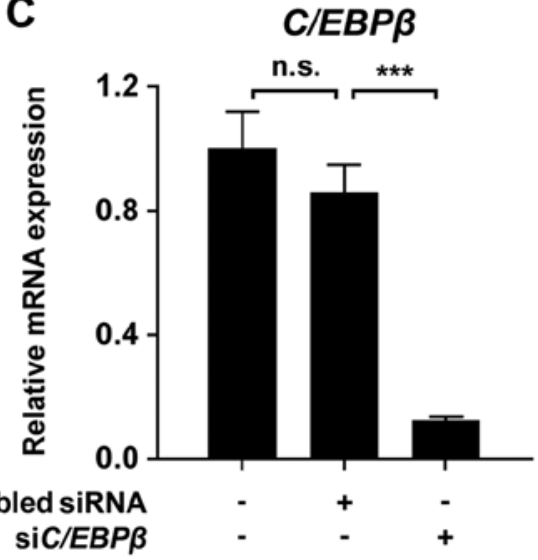

D

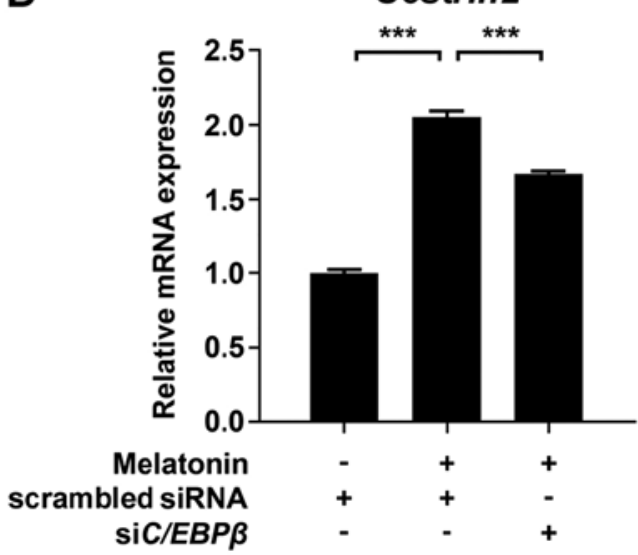

E

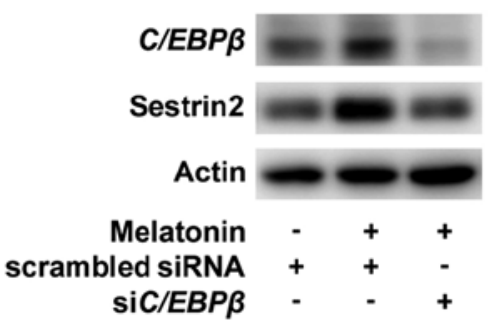

Figure 3. Effects of melatonin on Sestrin2 and C/EBP $\beta$ in VSMCs. Levels of (A) C/EBP $\beta$ and (B) Sestrin2 mRNAs in primary rat VSMCs treated with or without $2 \mathrm{mM}$ melatonin. (C-E) Primary rat VSMCs transfected with scrambled siRNA or siRNA targeting C/EBP $\beta$ (siC/EBP $\beta$ ) for 24 h were serum-starved for $24 \mathrm{~h}$, and then incubated with $10 \%$ FBS with or without $2 \mathrm{mM}$ melatonin. Levels of (C) C/EBP $\beta$ and (D) Sestrin2 mRNA and (E) C/EBP $\beta$ and Sestrin2 protein in VSMCs treated with or without $2 \mathrm{mM}$ melatonin. Data were normalized to the $36 B 4 \mathrm{mRNA}$ level, and expressed as the mean \pm SEM of three independent experiments. ${ }^{* *} \mathrm{P}<0.01 ;{ }^{* * *} \mathrm{P}<0.001$. n.s., not significant; VSMC, vascular smooth muscle cell; siRNA, small interfering RNA.

to suppression of VSMC proliferation. Furthermore, melatonin decreased VSMC apoptosis through inhibition of ROS accumulation by Sestrin2.

Although some previous studies reported that melatonin improves mitochondrial respiratory function and enhances ATP production, more recent studies showed that a supra-physiologic dose of melatonin can be harmful to the kidney and other tissues (11). Moreover, melatonin induces mitochondrial depolarization and decreases oxidative phosphorylation by inhibiting complex IV in some cancer cells $(20,21)$. In accordance with these findings, we also showed that melatonin increased mitochondrial energetic stress which was evident by decrease in basal OCR and maximal respiration, as well as ATP-linked respiration. Consequently, mitochondrial energetic stress by melatonin was responsible for induction of Sestrin2 in VSMCs.

Sestrin2 is an important regulator of mitohormesis in diverse tissues, including skeletal/cardiac muscle and brown adipose tissue (9). Previous study demonstrated that inhibitors of mitochondrial respiratory chain complex I and III induce transcription of Sestrin2 (22). Here, we showed that melatonin-induced Sestrin2 upregulation is mediated by $\mathrm{C} / \mathrm{EBP} \beta$, which is activated as an aspect of mitochondrial retrograde signaling in response to mitochondrial stress (23). Consistent with previous studies reporting Sestrin2-mediated inhibition of mTORC1 in various metabolic diseases, we also found that melatonin plays a critical role in limiting VSMC proliferation by the Sestrin $2 / m$ mORC 1 axis.

It should be noted that knockdown of Sestrin 2 promoted apoptosis in melatonin-treated VSMCs. Several lines of evidence show that apoptosis of VSMCs in atherosclerosis is sufficient to induce features of plaque vulnerability by thinning the fibrous cap and enlarging the necrotic core, ultimately resulting in plaque rupture (24). Accordingly, decreasing VSMC apoptosis could retard plaque size and instability, and thus has therapeutic potential (25). Sestrin2 promotes detoxification of ROS in two ways: By recycling peroxiredoxin as a part of an oxidoreductase enzyme, and by activating an antioxidant transcriptional program by stabilizing Nrf2 (6). In line with previous findings, we also observed that melatonin-induced Sestrin 2 prevents apoptosis of VSMCs by limiting excessive ROS accumulation. Therefore, melatonin represents a possible means to decrease both VSMC proliferation and apoptosis. Although it will be necessary to perform animal experiments to determine if these findings are valid in vivo, collectively, our results support previous studies showing that melatonin has anti-atherosclerotic effects in preclinical models $(26,27)$. 
A

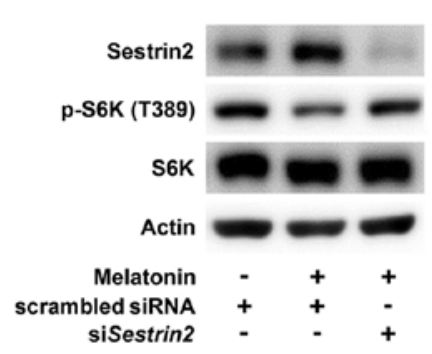

B

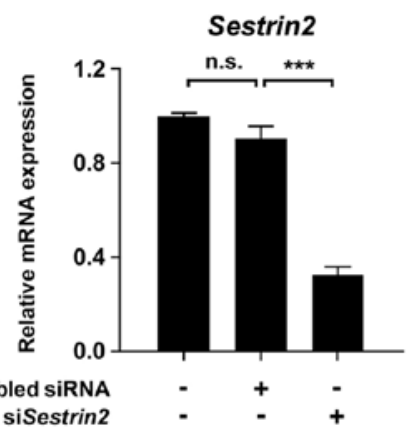

C

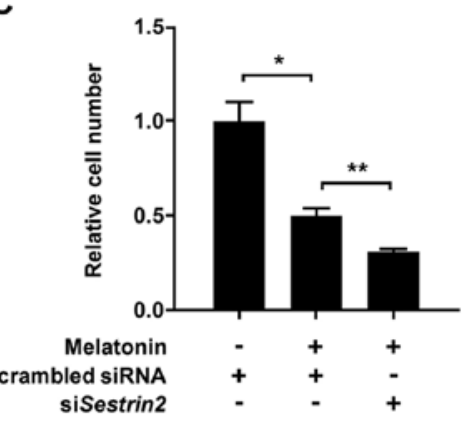

D
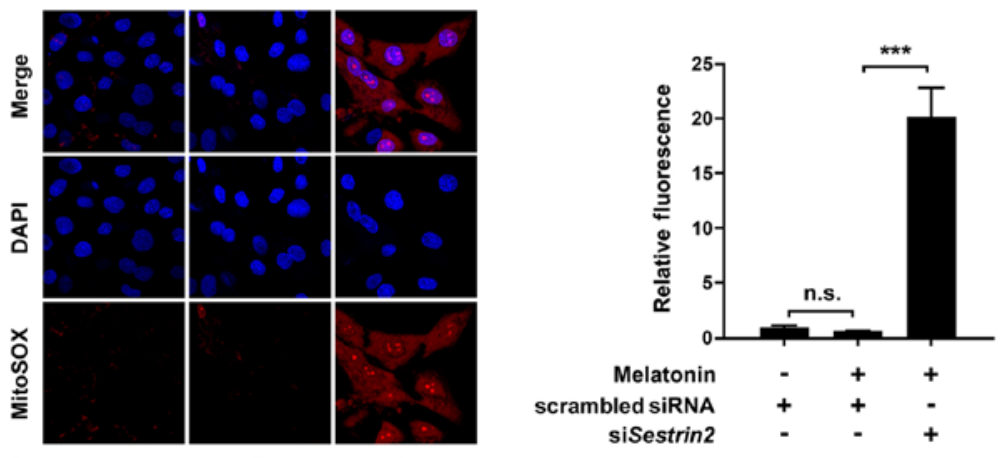

Melatonin
scrambled siRNA
siSestrin2

E

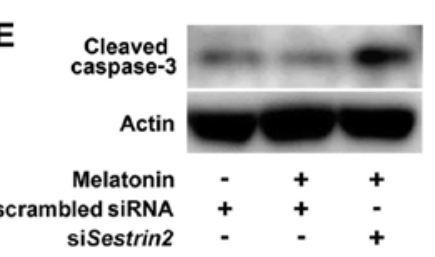

F

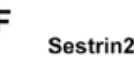

p-S6K (T389)
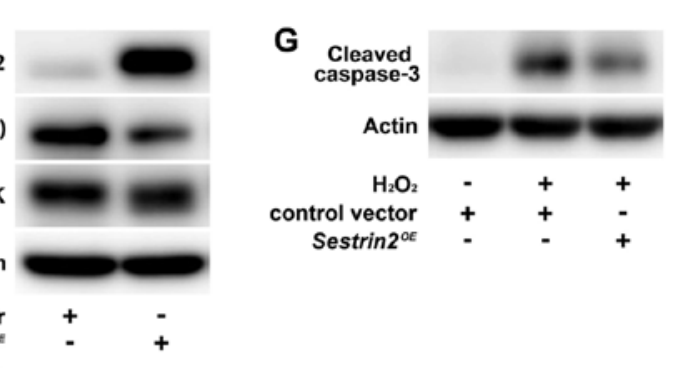

H

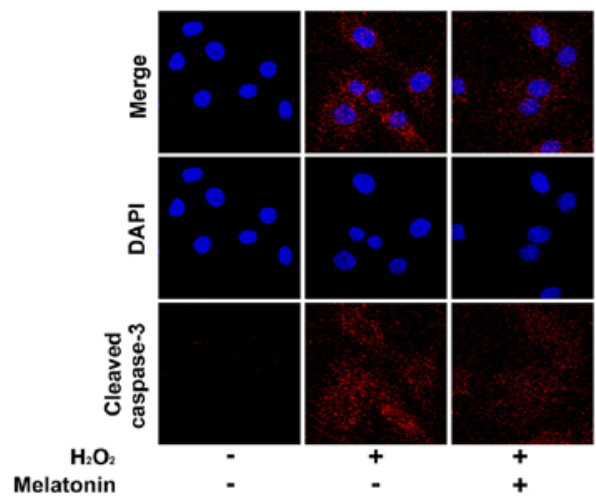

$+$

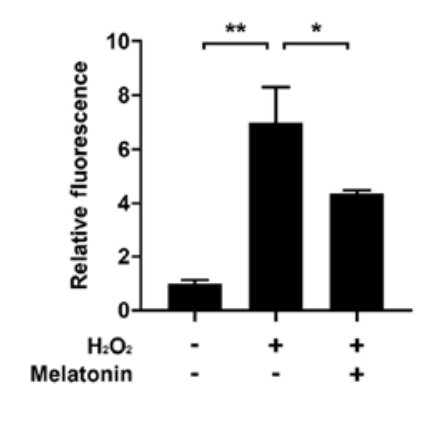

Figure 4. Effects of si-Sestrin2 on ROS generation and apoptosis in melatonin-treated VSMCs. Primary rat VSMCs transfected with scrambled siRNA or siSestrin 2 for $24 \mathrm{~h}$ were serum-starved for $24 \mathrm{~h}$, and then incubated with 10\% FBS with or without $2 \mathrm{mM}$ melatonin. Levels of (A) phosphorylated S6K (T389) and (B) Sesn 2 mRNA in VSMCs. (C) Relative VSMC number. Data are presented as the mean \pm SEM. (D) Representative fluorescence micrographs showing intracellular superoxide production in VSMCs. Bar graph shows quantitation of MitoSOX fluorescence intensity (red) with nuclear counterstaining with DAPI (blue), expressed as the mean \pm SEM. (E) Level of cleaved caspase 3 protein in VSMCs. (F and G) Primary rat VSMCs transfected with the Sestrin2-expression vector for $24 \mathrm{~h}$ were serum-starved for $24 \mathrm{~h}$, and then incubated with or without $1 \mathrm{mM} \mathrm{H}_{2} \mathrm{O}_{2}$ for $6 \mathrm{~h}$. (F) Phosphorylated S6K (T389) and (G) cleaved caspase 3 protein levels in VSMCs. (H) Primary rat VSMCs were pretreated with $2 \mathrm{mM}$ melatonin for $24 \mathrm{~h}$ and then treated with or without $1 \mathrm{mM} \mathrm{H} \mathrm{H}_{2} \mathrm{O}_{2}$ for $6 \mathrm{~h}$. Immunofluorescence images of cleaved caspase-3 (red) with nuclear counterstaining with DAPI (blue). Bar graphs show quantitation of cleaved caspase-3 immunofluorescence intensity, expressed as the mean \pm SEM. Magnification, $x 100 .{ }^{*} \mathrm{P}<0.05 ;{ }^{* *} \mathrm{P}<0.01 ;{ }^{* * *} \mathrm{P}<0.001$. n.s., not significant; VSMC, vascular smooth muscle cell; si/siRNA, small interfering RNA; ROS, reactive oxygen species.

In summary, we showed here that melatonin suppresses VSMC proliferation and apoptosis via Sestrin2-mediated inhibition of mTORC1 and ROS scavenging, respectively. Given its ability to control proliferation and apoptosis, melatonin represents a potential lead compound for prevention of vessel lumen constriction during the course of atherosclerosis and restenosis. Further studies should investigate how melatonin causes mitochondrial stress. 


\section{Acknowledgements}

Not applicable.

\section{Funding}

The present study was supported by the National Research Foundation of Korea (NRF) grants funded by the Ministry of Science and ICT (grant nos. NRF 2017M3A9G7073086 and NRF-2018R1A2A1A05077703), an NRF grant funded by the Ministry of Education (grant no. NRF-2017R1A6A3A04010231), and grants from the Korea Health Technology R\&D Project through the Korea Health Industry Development Institute funded by the Ministry of Health and Welfare, Republic of Korea (grant nos. HI16C1501 and HI15C0001).

\section{Availability of data and materials}

All data generated or analyzed during this study are included in this published article.

\section{Authors' contributions}

SHL and JKB conceptualized the study, designed the research and performed the experiments. MP performed the experiments. SWK, SWL, JGK, and IKL analyzed and interpreted the data. YKC and KGP designed the experiments, analyzed and interpreted the data, wrote and edited the manuscript, and supervised the project. All authors reviewed the data and provided feedback on the manuscript.

\section{Ethics approval and consent to participate}

The present study was approved by The Animal Care and Use Committee of Kyunpook National University School of Medicine (approval no. KNU-2011-0096-1).

\section{Patient consent for publication}

Not applicable.

\section{Competing interests}

The authors declare that they have no competing interests.

\section{References}

1. Sehgel NL, Vatner SF and Meininger GA: 'Smooth muscle cell stiffness syndrome'-Revisiting the structural basis of arterial stiffness. Front Physiol 6: 335, 2015.

2. Orford JL, Selwyn AP, Ganz P, Popma JJ and Rogers C: The comparative pathobiology of atherosclerosis and restenosis. Am J Cardiol 86: 6H-11H, 2000.

3. Bennett MR, Sinha S and Owens GK: Vascular smooth muscle cells in atherosclerosis. Circ Res 118: 692-702, 2016.

4. Rosner D, McCarthy N and Bennett M: Rapamycin inhibits human in stent restenosis vascular smooth muscle cells independently of pRB phosphorylation and p53. Cardiovasc Res 66: 601-610, 2005.

5. Martin KA, Rzucidlo EM, Merenick BL, Fingar DC, Brown DJ, Wagner RJ and Powell RJ: The mTOR/p70 S6K1 pathway regulates vascular smooth muscle cell differentiation. Am J Physiol Cell Physiol 286: C507-C517, 2004.

6. Lee JH, Budanov AV and Karin M: Sestrins orchestrate cellular metabolism to attenuate aging. Cell Metab 18: 792-801, 2013.
7. Budanov AV and Karin M: p53 target genes sestrin1 and sestrin2 connect genotoxic stress and mTOR signaling. Cell 134: 451-460, 2008.

8. Peng M, Yin N and Li MO: Sestrins function as guanine nucleotide dissociation inhibitors for Rag GTPases to control mTORC1 signaling. Cell 159: 122-133, 2014.

9. Ro SH, Semple I, Ho A, Park HW and Lee JH: Sestrin2, a regulator of thermogenesis and Mitohormesis in brown adipose tissue. Front Endocrinol (Lausanne) 6: 114, 2015.

10. Lee JH, Budanov AV, Talukdar S, Park EJ, Park HL, Park HW, Bandyopadhyay G, Li N, Aghajan M, Jang I, et al: Maintenance of metabolic homeostasis by Sestrin2 and Sestrin3. Cell Metab 16: 311-321, 2012.

11. Luchetti F, Canonico B, Betti M, Arcangeletti M, Pilolli F, Piroddi M, Canesi L, Papa S and Galli F: Melatonin signaling and cell protection function. FASEB J 24: 3603-3624, 2010.

12. Favero G, Moretti E, Bonomini F, Reiter RJ, Rodella LF and Rezzani R: Promising antineoplastic actions of melatonin. Front Pharmacol 9: 1086, 2018.

13. Zhao X, Sun J, Su W, Shan H, Zhang B, Wang Y, Shabanova A, Shan $\mathrm{H}$ and Liang $\mathrm{H}$ : Melatonin protects against lung fibrosis by regulating the Hippo/YAP pathway. Int J Mol Sci 19: pii: E1118, 2018.

14. Livak KJ and Schmittgen TD: Analysis of relative gene expression data using real-time quantitative PCR and the 2(-Delta Delta C(T)) method. Methods 25: 402-408, 2001.

15. Seo K, Ki SH and Shin SM: Sestrin2-AMPK activation protects mitochondrial function against glucose deprivation-induced cytotoxicity. Cell Signal 27: 1533-1543, 2015.

16. Zhao Q, Wang J, Levichkin IV, Stasinopoulos S, Ryan MT and Hoogenraad NJ: A mitochondrial specific stress response in mammalian cells. EMBO J 21: 4411-4419, 2002.

17. Parmigiani A, Nourbakhsh A, Ding B, Wang W, Kim YC Akopiants K, Guan KL, Karin M and Budanov AV: Sestrins inhibit mTORC1 kinase activation through the GATOR complex. Cell Rep 9: 1281-1291, 2014.

18. Saxton RA and Sabatini DM: mTOR Signaling in Growth, metabolism and disease. Cell 168: 960-976, 2017.

19. Budanov AV, Sablina AA, Feinstein E, Koonin EV and Chumakov PM: Regeneration of peroxiredoxins by p53-regulated sestrins, homologs of bacterial AhpD. Science 304: 596-600, 2004.

20. Loureiro R, Magalhaes-Novais S, Mesquita KA, Baldeiras I, Sousa IS, Tavares LC, Barbosa IA, Oliveira PJ and Vega-Naredo I: Melatonin antiproliferative effects require active mitochondrial function in embryonal carcinoma cells. Oncotarget 6: 17081-17096, 2015.

21. Sarti P, Magnifico MC, Altieri F, Mastronicola D and Arese M: New evidence for cross talk between melatonin and mitochondria mediated by a circadian-compatible interaction with nitric oxide. Int J Mol Sci 14: 11259-11276, 2013.

22. Garaeva AA, Kovaleva IE, Chumakov PM and Evstafieva AG: Mitochondrial dysfunction induces SESN2 gene expression through activating transcription Factor 4. Cell Cycle 15: 64-71, 2016.

23. Biswas G, Guha M and Avadhani NG: Mitochondria-to-nucleus stress signaling in mammalian cells: Nature of nuclear gene targets, transcription regulation, and induced resistance to apoptosis. Gene 354: 132-139, 2005.

24. Clarke MC, Figg N, Maguire JJ, Davenport AP, Goddard M, Littlewood TD and Bennett MR: Apoptosis of vascular smooth muscle cells induces features of plaque vulnerability in atherosclerosis. Nat Med 12: 1075-1080, 2006.

25. Lyon CA, Johnson JL, Williams H, Sala-Newby GB and George SJ: Soluble N-cadherin overexpression reduces features of atherosclerotic plaque instability. Arterioscler Thromb Vasc Biol 29: 195-201, 2009.

26. Cheng X, Wan Y, Xu Y, Zhou Q, Wang Y and Zhu H: Melatonin alleviates myosin light chain kinase expression and activity via the mitogen-activated protein kinase pathway during atherosclerosis in rabbits. Mol Med Rep 11: 99-104, 2015.

27. Hu ZP, Fang XL, Fang N, Wang XB, Qian HY, Cao Z, Cheng Y, Wang BN and Wang Y: Melatonin ameliorates vascular endothelial dysfunction, inflammation, and atherosclerosis by suppressing the TLR4/NF- $\kappa \mathrm{B}$ system in high-fat-fed rabbits. J Pineal Res 55: 388-398, 2013.

This work is licensed under a Creative Commons Attribution-NonCommercial-NoDerivatives 4.0 International (CC BY-NC-ND 4.0) License. 